\title{
The effectiveness of foot reflexology and behavior treatment in patient with overactive bladder syndrome: $\mathbf{A}$ \\ case report
}

\author{
Yasemin Aydin ${ }^{1}$
}

\begin{abstract}
This study was conducted in order to determine the foot reflexology and activity of behavior therapy in the patients who have overactive bladder syndrome.

In the study, behavior therapy (Bladder Training + Diet Modifications) and foot reflexology were applied together. Foot reflexology treatment was performed for 12 sessions and each session took 45 to 60 minutes to complete. For data collection, a descriptive information form, The Incontinence Impact Questionnaire (IIQ-7), Urinary Diaries and The 24 Hour Pad Test were administered. Assessments were done in two steps (prior to treatment and 3 months after treatment).

The primary outcome measure was the change in the diurnal micturition frequency. It was found that urgency and urgency incontinence episodes were significantly lower after treatment. According to study findings, foot reflexology, which was added to behavior therapy (Bladder Training + Diet Modifications), reduced most of urinary symptoms and affected quality of life positively in women with overactive bladder complaints.
\end{abstract}

Keywords: Overactive Bladder Syndrome; Foot Reflexology; Urinary Symptoms; Life Quality; Behavior Therapy.

\section{Introduction}

Overactive Bladder Syndrome (OABS), defined as "with or without Urgency Incontinence, existence of generally increased thamuria (frequency), need for urinate (urgency) and urinating at night (nocturia) complaints together" [1]. OABS syndrome is a disease that affects millions of people worldwide and has high prevalence. In NOBLE study (National Overactive Bladder Evaluation), OABS prevalence in USA stated as, $16 \%$ among man and 16.9 \% among women [2]. OABS syndrome does not threat the women life directly, however, it is such a problem that affects women's family and social life in physical and physiological aspects and as a result, it must be approached and evaluated carefully [3].

In the recent years, in protection and development of health, besides modern medicine, an increase was seen in methods which are named as complementary and alternative therapies (CAT). Alternative therapies is used for decrease disease and pain symptoms with nature's healing power and reinforce body's recuperative power and immune system [4]. As one of these methods, reflexology is commonly used, a non-invasive technique [5].

Reflexology, defined as "a technique that applied with hands on hands, foots and ears which are related to all glands, organs and body parts and helps normalization of body functions" by the International Reflexology Institute [6]. This method solves the blocked energy in the definite areas of body and puts self-therapy power in action. With the compulsion of sensory nerves, impulses go

1 Research Asistant, Ph.D,, Department of Midwifery, School of Health Sciences, Sakarya University, yaseminaydin@sakarya.edu.tr 
Aydın, Y. (2016). The effectiveness of foot reflexology and behavior treatment in patient with overactive bladder syndrome: A case report. Journal of Human Sciences, 13(3), 4446-4452. doi:10.14687/jhs.v13i3.4068

to brain and with the distribution of significant chemical substances, health and wellness are preserved. At the same time, blood stream fastens and homeostasis of the body provided. In addition, it helps the re-absorption of calcium, lactate, urine acid crystals [5].

When the effect of reflexology on overactive bladder examined, massage that applied on kidney and bladder with target organ effect, it increases perfusion and lowers muscle power and tonus, consequently, improvement on urinary symptoms was determined $[7,8]$. Because of its effects which can be harmful has not been reported in the literature, it was stated that reflexology can be used as a non-invasive procedure in treatment of OABS which is a multidimensional problem. Therefore, this study was conducted to determine the effectiveness of reflexology in women with OABS.

\section{Case Report}

56 years old woman patient is literate and housewife. It was determined that the patient who is 1 . Degree obese has chronic hypertension for five years and 8 years of asthma history and went through tonsillectomy operation in 1999 and appendectomy operation in 2007. When the drugs that patient use were examined, Seredite Discus $500 \mathrm{mcq}$, Coveram $10 \mathrm{mg}$ and Singulair 10 $\mathrm{mg}$ use was determined. The case in natural climacteric and has been through three spontaneous vaginal birth and when the lineage history examined, breast cancer in mother and Alzheimer history were determined. It was determined that patient's continuous complaints like continuous pad usage as a result of frequent urination, bladder pain from time to time, urinary urgency, urinary incontinence, urinary miss and waking up wet in nights were existed for 4 years. Patient's medical pre-diagnosis is overactive bladder syndrome and it was determined that patient received bladder training 1.5 years ago and biofeedback therapy and for six months, she used $1 \times 4 \mathrm{mg}$ Toviaz tb but because of the side effects, she quitted using the drug.

\section{Study Design}

After clinic and physical evaluation of the administrated patient, reflexology treatment, dietary modifications and bladder training were decided for application. Before the reflexology treatment application, Urinary Incontinence Impact Questionnaire (IIQ-7), urinary dairy, complete urine analysis and 24 hours pad test were applied. In order to measure the short term effect of the treatment, 3 months after the completion of bladder training, dietary modifications and foot reflexology, urinary dairy, IIQ-7 form applied again and 24 hours pad test was repeated.

\section{Data Collection}

Forms that used for data collection; Urinary Incontinence Impact Questionnaire, is a multidimensional question form that developed in order to determine urinary incontinence effect on life quality and one can answer all the form by oneself. Low points that were taken from IIQ-7 form show an increase in life quality. IIQ-7's validity and reliability work was done in 2007, locally [9].In the study, according to Incontinence Impact Questionnaire's measurement time, before treatment internal consistency coefficient Cronbach Alpha was found 0,890 and in the third month after treatment it was found as 0,920 .

Urinary diary, in order to evaluate urinary functions of the case, 24 hours urinary dairy was requested for completion. In consecutive four days, filled total 4 dairies were taken. In this diary, received liquid amounts, urinate necessity, urination hours in day time and missing episodes were requested to save in diary. Case, during the completion time of the dairy, was warned for not to change daily urination habits and received liquid amounts. With the data from diary, bladder training program of patient was edited [10,11]. Urinary diary was applied one day before the treatment start and after completion of the treatment, in the third month.

24 hours pad test was applied to patient one day before the treatment start and 3 months later after the completion of treatment. Pads that brought with leak proof bags were weighted with precision scale by the same researcher and results were saved in accordance with International 
Aydın, Y. (2016). The effectiveness of foot reflexology and behavior treatment in patient with overactive bladder syndrome: A case report. Journal of Human Sciences, 13(3), 4446-4452. doi:10.14687/jhs.v13i3.4068

Incontinence Union evaluation scales. According to this, classification represented as; 0-3 gr: negative, 4-20 gr: light, 21-74 gr: medium and 75 gr and over; severe urinary incontinence [12,13]. Behavior therapy was applied together with bladder training and dietary modification. In order to success the behavior therapy, it lasted at least 6 weeks [11,14].

Bladder training, in order to control the bladder and increase the capacity of bladder, was applied to case for 6 weeks. Before bladder training, urinary dairy was applied and patient's training program was edited in accordance with the diary. During the bladder training, liquid amount that was taken by patient followed, suggested liquid amount was wanted for consuming. From the urinary dairies, most frequent urination interval was determined. After this urination interval test, case was requested for extending urination interval half an hour. When the case accomplished the target, every week, case again was requested for extending the interval half an hour. If could not accomplish the target, interval decreased or remained same [11,14].

Dietary modifications were done by looking urinary diary of the patient. Consumption of adequate liquid and fibrous food was taken in consideration. Spicy, acidic and carbonated foods and any food and drinks with caffeine that can irritate bladder were limited. Foods that affects bladder also consumption of alcohol, tomato, honey and sugary foods, synthetic sweeteners, acidic drinks and fruits were taken in control [11,14].

Researchers implemented the reflexology method by completing the training program of reflexology implementations (100 hours of courses) which is approved by the Ministry of Education. Implementation steps;

- Provide information about the procedure to the patient

- Women are given the supine position and feet were slightly elevated by placing rolled towel under the feet.

- The application is done with bare hands.

- Implementation of reflexology starts from left foot. It is continued to be up to down.

- All implementations made to the left foot are made to right foot again.

- First 5-10 minutes, heat and relaxation movements are made on foot. Relaxation is provided by light effleurage, deep effleurage, rotation and stretching the Achilles movements.

- The first implementation is made to the "solar plexus" point in order to send message to whole body. The implementation is in the form of pressing and lifting the thumb 3 times. While the person is taking breath, pressure is applied to solar plexus, and while the person giving breath the pressure is removed. After 1 minute the pressure is applied to solar plexus.

- To brain area, six minutes massage is performed. In brain area; in thumb to pituitary gland, hypothalamus, brain and pineal body, inside of the food from thumb to middle of the heel to medulla spinalis the massage is performed.

- For 3 minutes, implementation is made to spleen and thyroid areas.

- For 3 minutes, implementation is made to spine area (L1-L5, T10-T12).

- For 3 minutes, massage is done to the lungs area.

- For 3 minutes, massage is done to diaphragm area.

- For 3 minutes, massage is done to stomach area.

- For 3 minutes, massage is done to adrenal glands area.

- For 3 minutes, massage is done to kidney area.

- For 3 minutes, massage is done to ureter area.

- For 10 minutes, massage is done to bladder area.

- For 3 minutes, implementation is performed to uterus, vagina, ovaries and fallopian tubes area. 
- It is recommended to drink plenty of water to accelerate the excretion of toxins after implementation $[16,17,18]$.

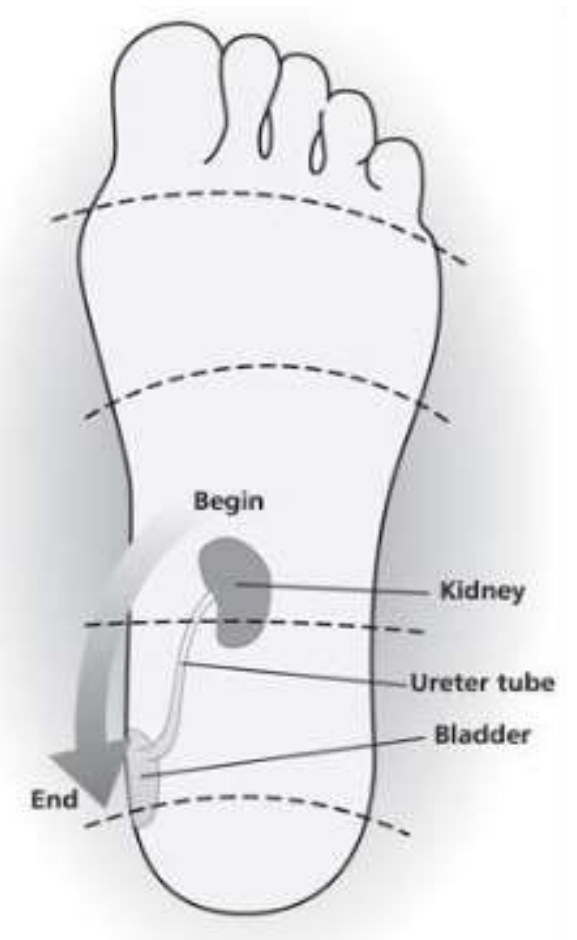

Figure 1. Urinary system reflex points

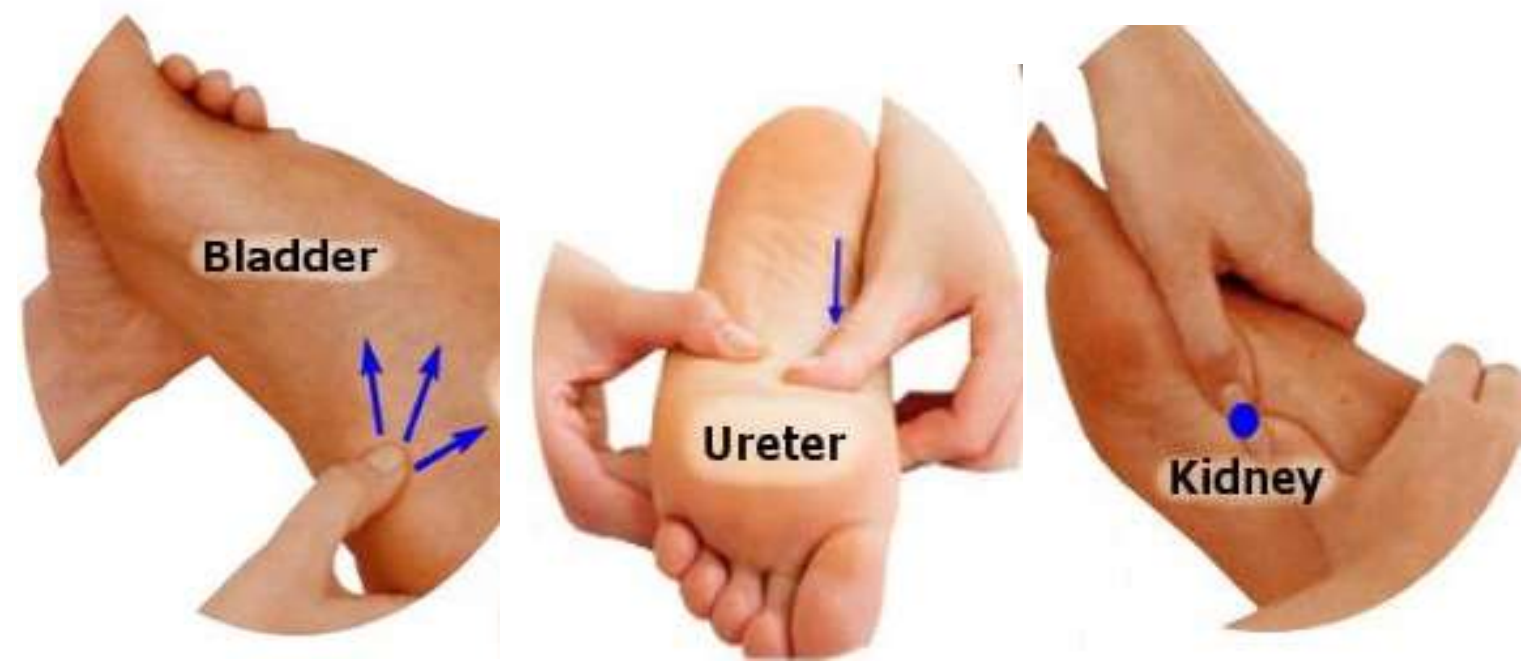

Figure 2. Reflexology points in Kidney, Ureter and Bladder Areas

\section{Ethical Considerations}

Written Permission has been taken from the hospital's research committee in order to conduct this research.

\section{Result and Discussion}

This study was done in order to determine the activity of behavior therapy that applied together with foot reflexology on the patient who has OABS. Hence, in the literature, it was seen that there are limited studies which evaluate the activity of reflexology, exist. In the study of Mak 
Aydin, Y. (2016). The effectiveness of foot reflexology and behavior treatment in patient with overactive bladder syndrome: A case report. Journal of Human Sciences, 13(3), 4446-4452. doi:10.14687/jhs.v13i3.4068

and friends; 54 women who have overactive bladder received reflexology, 43 women received nonspecific foot massage. In groups, 24 hours micturition frequency and urgency incontinence, incontinence complaints were decreased and in terms of 24 hours micturition frequency, it was determined that reflexology is more effective than the foot massage [8]. In the randomize controlled study of SievNer and friends; reflexology was applied to Multiple Skleroz patients and in the group that received reflexology, it was determined that urinary symptoms decreased significantly [7], again in Hughes and friends study which was conducted with Multiple Skleroz patients, reflexology and placebo foot massage were applied to patients for ten weeks and in the evaluation results, it was determined that there was 33\% improvement in muscular spasm and urgency symptoms [19].

In our study, likewise, complete urinary analysis of patient who was pre diagnosed with overactive bladder, was done and the result came out negative. Result of pad test which was applied before treatment was $77 \mathrm{gr}$ (Severe) and after reflexology and behavior therapy (bladder training, dietary modifications) it was observed that pad test result was decreased to $28 \mathrm{gr}$ (medium level). When urinary diary of patient was taken in evaluation, before treatment, urgency episodes 10,25, after treatment it decreased to 5,75 and daily urinary frequency was 14,25 , after treatment it decreased to 4,25 and lastly, urinary incontinence episodes lowered to 0,50 from 3,75.

Table 1 : Evaluation Parameters of Case Before and 3 month After the Treatment

\begin{tabular}{|l|l|l|}
\hline & Before Treatment & 3 Mo After Treatment \\
\hline $\begin{array}{l}\text { Pad } \\
\text { Test (gr) }\end{array}$ & 77 (Severe) & 28 (Medium Level) \\
\hline \multirow{4}{*}{ Urinary Diaries } & Daily Fluid Intake: $1250 \mathrm{ml}$ & Daily Fluid Intake: $2100 \mathrm{ml}$ \\
\cline { 2 - 3 } & Urgency: 10,25 & Urgency: 4,25 \\
\cline { 2 - 3 } & Urinary Frequency: 14,25 & Urinary Frequency: 5,75 \\
\cline { 2 - 3 } & Nocturia: 0,75 & Nocturia: 0,75 \\
\cline { 2 - 3 } & Urgency Incontinence: 3,75 & Urgency Incontinence: 0,50 \\
\hline
\end{tabular}

When the effects of urinary complaints on life quality was evaluated, IIQ-7 point average was high (19 points) as a result, before treatment life quality was affected negatively, and after treatment, increase in life quality was determined. 
Aydın, Y. (2016). The effectiveness of foot reflexology and behavior treatment in patient with overactive bladder syndrome: A case report. Journal of Human Sciences, 13(3), 4446-4452. doi:10.14687/jhs.v13i3.4068

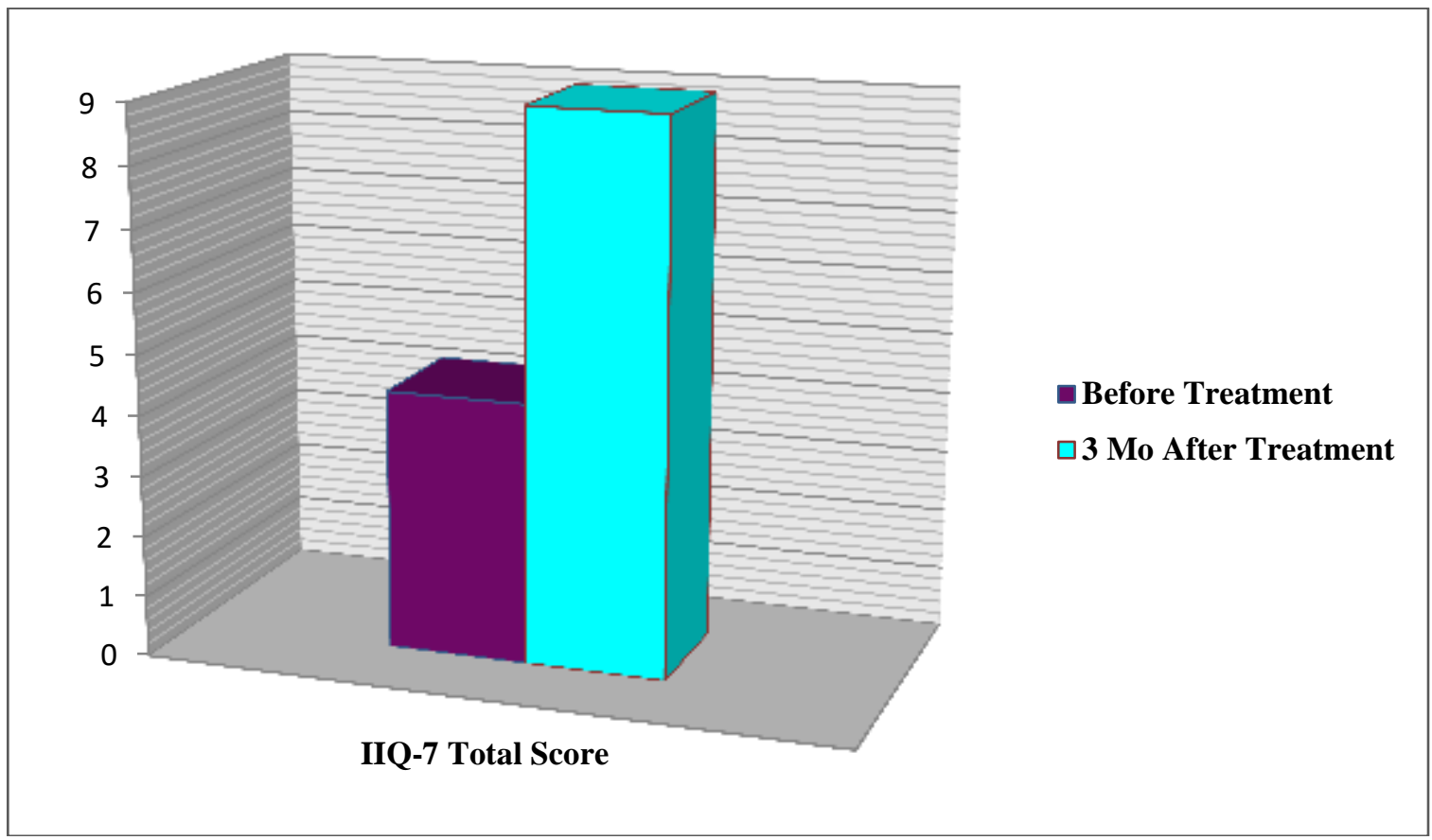

Figure 3. IIQ-7 Scores of Case Before and 3 month After the Treatment

\section{Conclusions and Recommendations}

As a result, in this case, it can be said that joint application of reflexology, dietary modifications and bladder training, lower urinary symptoms, therefore, life quality was increased. Hence, it was stated with studies that joint applications of behavior therapies that consist of bladder training, dietary modifications, lowers urgency, frequent urination, frequency and urge incontinence episodes [20,21]. In this study, it can be said that reflexology with the target organ effect, increases the perfusion of the massage which was applied on kidney and bladder area, lowers muscle power and tonus and in parallel, there were improvement in the symptoms. A search for new treatment modalities aimed to improve disturbing symptoms in OABS continues. It is of interest to note such positive effect of single intervention on a broad range of symptoms. This may possibly stem from the holistic approach of the reflexology (similarly to other complementary therapies), that treats the whole person rather than specific symptoms. In this direction, in OABS treatment, it can be suggested that health professionals should increase awareness about complementary and alternative approaches. Further clinical and laboratory studies are needed to validate these results and to understand the mechanisms by which reflexology improves symptoms secondary to OABS

\section{References}

1) Haylen BT, De Ridder D, Freeman RM, Swift SE, Berghmans B, Lee J, et al. (2010). International Urogynecological Association (IUGA)/International Continence Society (ICS) Joint Report on the Terminology for Female Pelvic Floor Dysfunction. Neurourologyand Urodynamics. 29: 4-20.

2) Stewart WF, Van Rooyen JB, Cundiff GW, Abrams P, Herzog AR, Corey R, Hunt TL, Wein AJ. (2003). Prevalence and Burden of Overactive Bladder in the United States. World J Urol. 20:327-36.

3) Lai H, Gardner V, Vetter J, Andriole GL. (2015). Correlation Between Psychological Stres Levels and The Severity of Overactive Bladder Symptoms. BMC Urol. 8;15(1):14. 

syndrome: A case report. Journal of Human Sciences, 13(3), 4446-4452. doi:10.14687/jhs.v13i3.4068

4) Lee J, Han M, Chung Y, Kim J, Choi J. (2011). Effects of Foot Reflexology on Fatigue, Sleep and Pain: A Systematic Review and Meta-Analysis. Journal of Korean Academy of Nursing. 41(6): 821-833.

5) Wang MY, Tsai PS, Lee P. H et al. (2008). The Efficacy of Reflexology: Systematic Review. Journal of Advanced Nursing. 62(5): 512- 520.

6) Vennells DF. (Çev. Soner, S.) (2004). Refleksoloji. Ege Mata Yayınları, II. Baskı, İzmir.

7) Siev-Ner DG, Lerner-Gevaand AA. Reflexology Treatment Relieves Symptoms of Multiple Sclerosis: A Randomized Controlled Study. Mult Scler August 2003; 9: 356-361,

8) Mak HL, Cheon WC, Wong T, Liu YS, TongWM. (2007). Randomized Controlled Trial of Foot Reflexology for Patients with Symptomatic Idiopathic Detrusor Overactivity. Int Urogynecol J Pelvic Floor Dysfunct. 18(6):653-8.

9) Çam C, Sakalli M, Ay P, Cam M, Karateke A. (2007). Validation of the Short Forms Of The Incontinence Impact Questionnaire (IIQ-7) and The Urogenital Distress Inventory (UDI-6) in a Turkish Population. Neurourol Urodyn. 26(1):129-33.

10) Thüroff JP, Abrams KE, Andersson W, Artibani E, Chartier-Kastler C, Hampel PH, et al. (2011). Guidelines on Urinary Incontinence, European Association of Urology. Eur Urol. 59(3): 387-400.

11) Lucas MG, Bedretdinova D, Bosch JLHR, Burkhard F, Cruz F, Nambiar AK, Nilsson CG, De Ridder DJMK, Tubaro A, Pickard RS. (2014). Guidelines on Urinary Incontinence. European Association of Urology.

12) Sullivan RO, Karantanis E, Stevermuer TL, Allen W, Moorea KH. (2004). Definition of Mild, Moderate and Severe Incontinence on the 24-Hour Pad Test. BJOG. 111: 859862.

13) Krhut J, Zachoval R, PP, Rosier PF, Valanský L, Martan A, Zvara P. (2014). Pad Weight Testing in The Evaluation of Urinary Incontinence. Neurourol Urodyn. 33(5):507-10.

14) NICE (National Institute for Health and Care Excellence). Overactive Bladder. http://pathways.nice.org.uk/pathways/urinary-incontinence-in-women/overactive bladder. (Erişim: 12. 01.2016).

15) Gala DR, Gala D, Gala S. (2014). Be Your Own Doctor with Foot Reflexology. Navneet Publications. India.

16) Goosmann L, Astrid I. (2010). Reflex Foot Massage Points. Tehran.

17) Gunnarsdottir TJ, Peden-McAlpine C. (2010). Effects of Reflexology On Fibromyalgia Symptoms: A Multiple Case Study. Complement Ther Clin Pract. 16(3):167-72.

18) Hughes CM, Smyth S, Lowe-Strong AS. (2009). Reflexology for the Treatment of Pain in People with Multiple Sclerosis: A Double-blind Randomised Sham-Controlled Clinical Trial, Mult Scler. 15: 1329.

19) Olivera CK, Meriwether K, El-Nashar S, et al. (2016). Non-Antimuscarinic Treatment For Overactive Bladder: A Systematic Review. Am J Obstet Gynecol. Doi: 10.1016/J.Ajog.2016.01.156.

20) Azuri J, Kafri R, Ziv-Baran T, Stav K. (2016). Outcomes of Different Protocols Of Pelvic Floor Physical Therapy And Anti-Cholinergics in Women With Wet Overactive Bladder: A 4-Year Follow-Up. Neurourol Urodyn. doi: 10.1002/nau.23016. 